This is a postprint version of the following published document:

Koch, Tobias. The Shannon lower bound is asymptotically tight. IEEE transactions on information theory, 62(11), pp. 6155-6161, November 2016

DOI: https://doi.org/10.1109/TIT.2016.2604254

C2016 IEEE. Personal use of this material is permitted. Permission from IEEE must be obtained for all other uses, in any current or future media, including reprinting/republishing this material for advertising or promotional purposes, creating new collective works, for resale or redistribution to servers or lists, or reuse of any copyrighted component of this work in other works. 


\title{
The Shannon Lower Bound is Asymptotically Tight
}

\author{
Tobias Koch, Member, IEEE
}

\begin{abstract}
The Shannon lower bound is one of the few lower bounds on the rate-distortion function that holds for a large class of sources. In this paper, which considers exclusively normbased difference distortion measures, it is demonstrated that its gap to the rate-distortion function vanishes as the allowed distortion tends to zero for all sources having finite differential entropy and whose integer part has finite entropy. Conversely, it is demonstrated that if the integer part of the source has infinite entropy, then its rate-distortion function is infinite for every finite distortion level. Thus, the Shannon lower bound provides an asymptotically tight bound on the rate-distortion function if, and only if, the integer part of the source has finite entropy.
\end{abstract}

Index Terms-Rate-distortion theory, Rényi information dimension, Shannon lower bound.

\section{INTRODUCTION}

$\mathbf{S}$ UPPOSE that we wish to quantize a memoryless, $d$ dimensional source with a distortion not larger than $D$. More specifically, suppose a source produces the sequence of independent and identically distributed (i.i.d.), $d$-dimensional, real-valued, random vectors $\left\{\mathbf{X}_{k}, k \in \mathbb{Z}\right\}$ (where $\mathbb{Z}$ denotes the set of integers) according to the distribution $P_{\mathbf{X}}$, and suppose that we employ a vector quantizer that produces a sequence of reconstruction vectors $\left\{\hat{\mathbf{X}}_{k}, k \in \mathbb{Z}\right\}$ satisfying

$$
\varlimsup_{n \rightarrow \infty} \frac{1}{n} \sum_{k=1}^{n} \mathrm{E}\left[\left\|\mathbf{X}_{k}-\hat{\mathbf{X}}_{k}\right\|^{r}\right] \leq D
$$

for some norm $\|\cdot\|$ and some $r>0$. (We use $\overline{l i m}$ to denote the limit superior and lim to denote the limit inferior.) Ratedistortion theory states that if for every blocklength $n$ and distortion constraint $D$ we quantize the sequence of source vectors $\mathbf{X}_{1}, \ldots, \mathbf{X}_{n}$ to one of $e^{n R(D)}$ possible sequences of reconstruction vectors $\hat{\mathbf{X}}_{1}, \ldots, \hat{\mathbf{X}}_{n}$, then the smallest rate $R(D)$ (in nats per source vector) for which there exists a vector quantizer satisfying (1) is given by [1], [2]

$$
R(D)=\inf _{P_{\hat{\mathbf{X}} \mid \mathbf{X}}: \mathrm{E}\left[\|\mathbf{X}-\hat{\mathbf{X}}\|^{r}\right] \leq D} I(\mathbf{X} ; \hat{\mathbf{X}})
$$

where the infimum is over all conditional distributions of $\hat{\mathbf{X}}$ given $\mathbf{X}$ for which

$$
\mathrm{E}\left[\|\mathbf{X}-\hat{\mathbf{X}}\|^{r}\right] \leq D
$$

T. Koch has been supported in part by a Marie Curie Career Integration Grant through the 7th European Union Framework Programme under Grant 333680, by the Ministerio de Economía of Spain under Grants TEC201341718-R, RYC-2014-16332, and TEC2015-69648-REDC, and by the Comunidad de Madrid under Grant S2013/ICE-2845. The material in this paper was presented in part at the 2016 International Zurich Seminar on Communications, Zurich, Switzerland, March 2016.

The author is with the Signal Theory and Communications Department, Universidad Carlos III de Madrid, 28911, Leganés, Spain and also with the Gregorio Marañón Health Research Institute, Madrid, Spain (e-mail: kochetsc.uc3m.es). and where the expectation in (3) is computed with respect to the joint distribution $P_{\mathbf{X}} P_{\hat{\mathbf{X}} \mid \mathbf{X}}$. Here and throughout the paper we omit the time indices where they are immaterial. The rate $R(D)$ as a function of $D$ is referred to as the rate-distortion function.

Unfortunately, the rate-distortion function is unknown except in a few special cases. It therefore needs to be assessed by means of upper and lower bounds. Arguably, for sources with a probability density function (pdf) and finite differential entropy $h(\mathbf{X})$, the most important lower bound is the Shannon lower bound [1], [2], which for a $d$-dimensional, real-valued source and the distortion constraint (3) is given by [3]

$$
\begin{aligned}
R_{\mathrm{SLB}}(D)= & h(\mathbf{X})+\frac{d}{r} \log \frac{1}{D} \\
& -\frac{d}{r} \log \left(\frac{r}{d}\left(V_{d} \Gamma(1+d / r)\right)^{r / d} e\right) .
\end{aligned}
$$

Here $\log (\cdot)$ denotes the natural $\operatorname{logarithm}, V_{d}$ denotes the volume of the $d$-dimensional unit ball $\left\{\mathbf{x} \in \mathbb{R}^{d}:\|\mathbf{x}\| \leq 1\right\}$, and $\Gamma(\cdot)$ denotes the Gamma function. While this lower bound is tight only for some special sources, it converges to the rate-distortion function as the allowed distortion $D$ tends to zero, provided that the source satisfies some regularity conditions; see, e.g., [4]-[7]. A finite-blocklength refinement of the Shannon lower bound has recently been given by Kostina [8], [9].

To the best of our knowledge, the most general proof of the asymptotic tightness of the Shannon lower bound is due to Linder and Zamir [7]. While Linder and Zamir considered more general distortion measures, specialized to the normbased distortion (3), they showed the following.

Theorem 1 (Linder and Zamir [7, Cor. 1]): Suppose that $\mathbf{X}$ has a pdf and that $h(\mathbf{X})$ is finite. Assume further that there exists an $\alpha>0$ such that $\mathbf{E}\left[\|\mathbf{X}\|^{\alpha}\right]<\infty$. Then the Shannon lower bound is asymptotically tight, i.e.,

$$
\lim _{D \downarrow 0}\left\{R(D)-R_{\mathrm{SLB}}(D)\right\}=0 .
$$

\section{Proof: See [7].}

The theorem's conditions are very mild and satisfied by the most common source distributions. In fact, Theorem 1 demonstrates that the Shannon lower bound provides a good approximation of the rate-distortion function for small distortions even if there exists no quantizer with a finite number of codevectors and of finite distortion, i.e., when $\mathrm{E}\left[\|\mathbf{X}\|^{r}\right]=\infty$. However, the theorem's conditions are more stringent than the ones sometimes encountered in analyses of the rate and distortion redundancies of high-resolution quantizers. This is relevant because the Shannon lower bound is often used as a benchmark against which the performance of such quantizers is measured. 
For example, Gish and Pierce [10] studied the smallest output entropy that can be achieved via scalar quantization with given expected quadratic distortion, i.e.,

$$
R_{\mathrm{s}}(D)=\inf _{q: \mathrm{E}\left[(X-q(X))^{2}\right] \leq D} H(q(X))
$$

where the infimum is over all deterministic mappings $q(\cdot)$ from the source alphabet $\mathcal{X}$ to some (countable) reconstruction alphabet $\hat{\mathcal{X}}$ satisfying $\mathrm{E}\left[(X-q(X))^{2}\right] \leq D$. For onedimensional sources that have a pdf satisfying some continuity and decay constraints, they showed that the asymptotic excess rate with respect to the rate-distortion function is given by

$$
\varliminf_{D \downarrow 0}\left\{R_{\mathrm{s}}(D)-R(D)\right\}=\frac{1}{2} \log \frac{\pi e}{6} .
$$

They further showed that this excess rate can be achieved by a uniform quantizer, hence the well-known result that "uniform quantizers are asymptotically optimal as the allowed distortion tends to zero." ${ }^{1}$ Since the rate-distortion function $R(D)$ is in general unknown, they showed instead that

$$
\underline{\lim }_{D \downarrow 0}\left\{R_{\mathrm{s}}(D)-R_{\mathrm{SLB}}(D)\right\}=\frac{1}{2} \log \frac{\pi e}{6} .
$$

This is equivalent to (7) whenever the Shannon lower bound is asymptotically tight.

A dual formulation of (7) was given by Zador [12] as the smallest asymptotic excess distortion with respect to the distortion-rate function as the rate tends to infinity. While Zador's original derivation was flawed, a rigorous proof of the same result was given by Gray, Linder, and Li [13]. In their work, they consider $d$-dimensional source vectors $\mathbf{X}$ that have a pdf, whose differential entropy is finite, and that satisfy

$$
H(\lfloor\mathbf{X}\rfloor)<\infty .
$$

Here $\lfloor\mathbf{a}\rfloor, \mathbf{a}=\left(a_{1}, \ldots, a_{d}\right) \in \mathbb{R}^{d}$ denotes the elementwise floor-function, i.e., $\lfloor\mathbf{a}\rfloor=\left(\left\lfloor a_{1}\right\rfloor, \ldots,\left\lfloor a_{d}\right\rfloor\right) \in \mathbb{Z}^{d}$ where $\left\lfloor a_{\ell}\right\rfloor$ denotes the largest integer not larger than $a_{\ell}$. In words, condition (9) demands that quantizing the source with a cubic lattice quantizer of unit-volume cells gives rise to a discrete random vector of finite entropy. This ensures that the quantizer output can be further compressed using a lossless variablelength code of finite expected length. Koch and Vazquez-Vilar [14], [15] recently demonstrated that these assumptions are also sufficient to recover Gish and Pierce's result (7).

As we shall argue below, (9) is weaker than the assumption $\mathrm{E}\left[\|\mathbf{X}\|^{\alpha}\right]<\infty$ required in Theorem 1 for the asymptotic tightness of the Shannon lower bound. One may thus wonder whether there are sources for which the performance of highresolution quantizers can be evaluated but the Shannon lower bound does not constitute a relevant performance benchmark. In this paper, we demonstrate that this is not the case. We show that for sources that have a pdf and whose differential entropy is finite, the Shannon lower bound (4) is asymptotically tight if (9) is satisfied. Conversely, we demonstrate that for sources

\footnotetext{
${ }^{1}$ The fact that, in the high-resolution case, the expected quadratic distortion of uniform scalar quantization exceeds the least distortion achievable by any quantization scheme whatsoever by a factor of only $\pi e / 6$ was already discovered by Koshelev in 1963. See [11] and references therein for more details.
}

that do not satisfy (9), the rate-distortion function is infinite for any finite distortion level. Hence, condition (9) is necessary and sufficient for the asymptotic tightness of the Shannon lower bound.

The quantity $H(\lfloor\mathbf{X}\rfloor)$ in (9) is intimately related with the Rényi information dimension [16], defined as

$$
d(\mathbf{X}) \triangleq \lim _{m \rightarrow \infty} \frac{H(\lfloor m \mathbf{X}\rfloor / m)}{\log m}, \quad \text { if the limit exists }
$$

which in turn coincides with the rate-distortion dimension introduced by Kawabata and Dembo [17]; see also [18]. Generalizing Proposition 1 in [18] to the vector case, it can be shown that the Rényi information dimension is finite if, and only if, (9) is satisfied and that a sufficient condition for finite Rényi information dimension is $\mathrm{E}[\log (1+\|\mathbf{X}\|)]<\infty$, which in turn holds for any source vector for which $E\left[\|\mathbf{X}\|^{\alpha}\right]<\infty$ for some $\alpha>0$. Thus, (9) is indeed weaker than the assumption that $\mathrm{E}\left[\|\mathbf{X}\|^{\alpha}\right]<\infty$.

It is common to assume that the differential entropy of the source is finite, since otherwise the Shannon lower bound (4) is uninteresting. We next briefly discuss how (9) and the assumption of a finite differential entropy are related. As demonstrated, e.g., in the proof of Theorem 3 in [19], a finite $H(\lfloor\mathbf{X}\rfloor)$ implies that $h(\mathbf{X})<\infty$. In fact, one can show that if (9) holds and the random vector $\mathbf{X}$ has a pdf, then $h(\mathbf{X}) \leq H(\lfloor\mathbf{X}\rfloor)[20$, Cor. 1]. Conversely, one can find sources for which the differential entropy is finite but $H(\lfloor\mathbf{X}\rfloor)$ is infinite. For example, consider a one-dimensional source with pdf

$$
f_{X}(x)=\sum_{m=2}^{\infty} p_{m} m \mathbf{1}\left\{m \leq x \leq m+\frac{1}{m}\right\}, \quad x \in \mathbb{R}
$$

where

$$
\begin{aligned}
p_{m} & =\frac{1}{\mathrm{~K} m \log ^{2} m}, \quad m=2,3, \ldots \\
\mathrm{K} & =\sum_{m=2}^{\infty} \frac{1}{m \log ^{2} m}
\end{aligned}
$$

and $\mathbf{1}\{\cdot\}$ denotes the indicator function. It is easy to check that for such a source

$$
\begin{aligned}
H(\lfloor X\rfloor) & =\sum_{m=2}^{\infty} p_{m} \log \frac{1}{p_{m}} \\
& =\sum_{m=2}^{\infty} \frac{\log \mathrm{K}+\log m+2 \log \log m}{\mathrm{~K} m \log ^{2} m} \\
& =\infty
\end{aligned}
$$

and

$$
\begin{aligned}
h(X) & =-\int f_{X}(x) \log f_{X}(x) \mathrm{d} x \\
& =\sum_{m=2}^{\infty} \frac{\log \mathrm{K}+2 \log \log m}{\mathrm{~K} m \log ^{2} m} \\
& <\infty .
\end{aligned}
$$

(See remark after Theorem 1 in [16, pp. 197-198].) Thus, for sources satisfying $h(\mathbf{X})>-\infty$, a finite $H(\lfloor\mathbf{X}\rfloor)$ implies a finite differential entropy but not vice versa. 


\section{Main Results}

The main results of this paper are presented in the following two theorems: Theorem 2 demonstrates that the gap between the Shannon lower bound and the rate-distortion function vanishes as the allowed distortion tends to zero for all sources having finite differential entropy and finite $H(\mid \mathbf{X}\rfloor)$. Theorem 3 demonstrates that if $H(\lfloor\mathbf{X}\rfloor)$ is infinite, then the rate-distortion function of $\mathbf{X}$ is infinite for every finite distortion level. These two results imply that, for sources with finite differential entropy, the Shannon lower bound is asymptotically tight if, and only if, $H(\lfloor\mathbf{X}\rfloor)$ is finite.

Theorem 2 (Asymptotic Tightness): Suppose that the $d$ dimensional, real-valued source $\mathbf{X}$ has a pdf. Further assume that $H(\mid \mathbf{X}\rfloor)<\infty$ and $|h(\mathbf{X})|<\infty$. Then the Shannon lower bound is asymptotically tight, i.e.,

$$
\lim _{D \downarrow 0}\left\{R(D)-R_{\mathrm{SLB}}(D)\right\}=0 .
$$

Proof: See Section III.

Theorem 3 (Finite Rate-Distortion Function): For every distortion level $D>0$, the rate-distortion function $R(D)$ of the $d$-dimensional, real-valued source $\mathbf{X}$ is finite if, and only if, $H(\lfloor\mathbf{X}\rfloor)<\infty$.

Proof: See Section IV.

In all fairness, we should mention that Linder and Zamir also presented conditions for the asymptotic tightness of the Shannon lower bound that are weaker than the ones presented in Theorem 1; see [7, Th. 1]. Specifically, they showed that the Shannon lower bound is asymptotically tight if $\mathbf{X}$ has a pdf, if $h(\mathbf{X})$ is finite, and if there exists a function $\delta: \mathbb{R}^{d} \rightarrow[0, \infty)$ satisfying the following: ${ }^{2}$

(i) For every $D>0$, the equations

$$
\begin{array}{r}
a(D) \int_{\mathbb{R}^{d}} e^{-s(D) \delta(\mathbf{x})} \mathrm{d} \mathbf{x}=1 \\
a(D) \int_{\mathbb{R}^{d}} \delta(\mathbf{x}) e^{-s(D) \delta(\mathbf{x})} \mathrm{d} \mathbf{x}=D
\end{array}
$$

have a unique pair of solutions $(a(D), s(D))$. Moreover, $a(D)$ and $s(D)$ are continuous functions of $D$.

(ii) Let $\mathbf{W}_{D}$ be a random vector with pdf

$$
\mathbf{x} \mapsto a(D) e^{-s(D) \delta(\mathbf{x})} .
$$

Then $\mathbf{W}_{D} \Rightarrow \mathbf{0}$ as $D \rightarrow 0$, where we use " $\Rightarrow$ " to denote convergence in distribution and $\mathbf{0}$ denotes the all-zero vector.

(iii) Let $\mathbf{Z}_{D}$ be a random vector that is independent of $\mathbf{X}$ and has pdf

$$
\mathbf{z} \mapsto\left(\frac{d}{r}\right)^{\frac{d}{r}-1} \frac{1}{V_{d} \Gamma(d / r) D^{\frac{d}{r}}} e^{-\frac{d}{r D}\|\mathbf{z}\|^{r}} .
$$

Then $\delta(\cdot)$ satisfies $0<\mathrm{E}[\delta(\mathbf{X})]<\infty$ and $\mathrm{E}\left[\delta\left(\mathbf{X}+\mathbf{Z}_{D}\right)\right]$ tends to $\mathbf{E}[\delta(\mathbf{X})]$ as $D$ tends to zero.

It is unclear whether there exists a function $\delta(\cdot)$ with the above properties that allows us to prove the asymptotic tightness of the Shannon lower bound for all source vectors

\footnotetext{
${ }^{2}$ These conditions correspond to the conditions in [7, Th. 1] where $\rho(\mathbf{x})=$ $\|\mathbf{x}\|^{r}, \mathbf{x} \in \mathbb{R}^{d}$
}

X satisfying $H(\lfloor\mathbf{X}\rfloor)<\infty$ and $|h(\mathbf{X})|<\infty$. In fact, even if there existed such a function, proving that it satisfies the required conditions may be complicated. Fortunately, the existence of such a function is not essential. Indeed, the proof of Theorem 2 follows closely the proof of Theorem 1 in [7] but avoids the use of $\delta(\cdot)$.

\section{PROOF OF THEOREM 2}

The first steps in our proof are identical to the ones in the proof of Theorem 1 in [7]. To keep this paper self-contained, we reproduce all the steps.

To prove asymptotic tightness of $R_{\mathrm{SLB}}(D)$, we derive an upper bound on $R(D)$ whose gap to $R_{\mathrm{SLB}}(D)$ vanishes as $D$ tends to zero. In view of (2), an upper bound on $R(D)$ follows by choosing $\hat{\mathbf{X}}=\mathbf{X}+\mathbf{Z}_{D}$, where $\mathbf{Z}_{D}$ is a $d$-dimensional, realvalued, random vector that is independent of $\mathbf{X}$ and has pdf (17). It can be shown that $\mathbf{Z}_{D}$ satisfies $\mathrm{E}\left[\left\|\mathbf{Z}_{D}\right\|^{r}\right]=D$; see, e.g., [3, Sec. VI]. It follows that

$$
\begin{aligned}
R(D) & \leq I\left(\mathbf{X} ; \mathbf{X}+\mathbf{Z}_{D}\right) \\
& =h\left(\mathbf{X}+\mathbf{Z}_{D}\right)-h\left(\mathbf{Z}_{D}\right) .
\end{aligned}
$$

Furthermore, by evaluating $h\left(\mathbf{Z}_{D}\right)$ and comparing the result with (4), we have

$$
R_{\mathrm{SLB}}(D)=h(\mathbf{X})-h\left(\mathbf{Z}_{D}\right) .
$$

Combining (18) and (19) gives

$$
0 \leq R(D)-R_{\mathrm{SLB}}(D) \leq h\left(\mathbf{X}+\mathbf{Z}_{D}\right)-h(\mathbf{X}) .
$$

Thus, asymptotic tightness of the Shannon lower bound follows by proving that

$$
\varlimsup_{D \downarrow 0} h\left(\mathbf{X}+\mathbf{Z}_{D}\right) \leq h(\mathbf{X}) .
$$

To this end, we follow the steps (17)-(21) in [7] but with $Y_{\Delta(D)}$ and $Y_{\Delta(0)}$ there replaced by the random vectors $\mathbf{Y}_{D}$ and $\mathbf{Y}_{0}$ having the respective pdfs

$$
\begin{aligned}
& f_{\mathbf{Y}_{D}}(\mathbf{y})=\sum_{\mathbf{i} \in \mathbb{Z}^{d}} \operatorname{Pr}\left(\left\lfloor\mathbf{X}+\mathbf{Z}_{D}\right\rfloor=\mathbf{i}\right) \mathbf{1}\{\lfloor\mathbf{y}\rfloor=\mathbf{i}\} \\
& f_{\mathbf{Y}_{0}}(\mathbf{y})=\sum_{\mathbf{i} \in \mathbb{Z}^{d}} \operatorname{Pr}(\lfloor\mathbf{X}\rfloor=\mathbf{i}) \mathbf{1}\{\lfloor\mathbf{y}\rfloor=\mathbf{i}\}
\end{aligned}
$$

for $\mathbf{y} \in \mathbb{R}^{d}$. It follows that

$$
D\left(f_{\mathbf{X}+\mathbf{Z}_{D}} \| f_{\mathbf{Y}_{D}}\right)=H\left(\left\lfloor\mathbf{X}+\mathbf{Z}_{D}\right\rfloor\right)-h\left(\mathbf{X}+\mathbf{Z}_{D}\right)
$$

and

$$
D\left(f_{\mathbf{X}} \| f_{\mathbf{Y}_{0}}\right)=H(\lfloor\mathbf{X}\rfloor)-h(\mathbf{X})
$$

where $D(f \| g)$ denotes the relative entropy between the pdfs $f$ and $g$ [21, Eq. (9.46)].

The random vector $\mathbf{Z}_{D}$ has the same pdf as $D^{1 / r} \mathbf{Z}_{1}$, where $\mathbf{Z}_{1}$ denotes $\mathbf{Z}_{D}$ for $D=1$. Consequently, $\mathbf{Z}_{D} \rightarrow \mathbf{0}$ almost surely as $D$ tends to zero and, hence, also in distribution. Since $\mathbf{X}$ and $\mathbf{Z}_{D}$ are independent, it follows that $\mathbf{X}+\mathbf{Z}_{D} \Rightarrow \mathbf{X}$ as $D$ tends to zero. Furthermore, since $\mathbf{X}$ has a pdf and the set $\mathbb{Z}^{d}$ is countable, the probability $\operatorname{Pr}\left(\mathbf{X} \in \mathbb{Z}^{d}\right)$ is zero, so [22, Th. 2.8.1, p. 122]

$$
\lim _{D \downarrow 0} \operatorname{Pr}\left(\left\lfloor\mathbf{X}+\mathbf{Z}_{D}\right\rfloor=\mathbf{i}\right)=\operatorname{Pr}(\lfloor\mathbf{X}\rfloor=\mathbf{i}), \quad \mathbf{i} \in \mathbb{Z}^{d} .
$$


We thus conclude that $f_{\mathbf{Y}_{D}}$ converges pointwise to $f_{\mathbf{Y}_{0}}$, which by Scheffe's lemma [23, Th. 16.12] implies that $\mathbf{Y}_{D} \Rightarrow \mathbf{Y}_{0}$ as $D$ tends to zero.

By the lower semicontinuity of relative entropy (see, e.g., the proof of Lemma 4 in [24] and references therein), it follows that

$$
\varliminf_{D \downarrow 0} D\left(f_{\mathbf{X}+\mathbf{z}_{D}} \| f_{\mathbf{Y}_{D}}\right) \geq D\left(f_{\mathbf{X}} \| f_{\mathbf{Y}_{0}}\right) .
$$

Together with (23) and (24), this yields

$$
\frac{\lim }{D \downarrow 0}\left\{H\left(\left\lfloor\mathbf{X}+\mathbf{Z}_{D}\right\rfloor\right)-h\left(\mathbf{X}+\mathbf{Z}_{D}\right)\right\} \geq H(\lfloor\mathbf{X}\rfloor)-h(\mathbf{X}) .
$$

Since $H(\lfloor\mathbf{X}\rfloor)<\infty$ and $|h(\mathbf{X})|<\infty$, the claim (21) follows from (27) by showing that $H\left(\left\lfloor\mathbf{X}+\mathbf{Z}_{D}\right\rfloor\right)$ tends to $H(\lfloor\mathbf{X}\rfloor)$ as $D$ tends to zero. To this end, we need the following lemma, which we state in its most general form since it may be of independent interest.

Lemma 1: Let $\mathbf{X}$ and $\mathbf{Z}$ be independent, $d$-dimensional, real-valued random vectors. Assume that $\mathrm{E}\left[\|\mathbf{Z}\|^{r}\right]<\infty$.

(i) If $H(\lfloor\mathbf{X}\rfloor)=\infty$, then $H(\lfloor\mathbf{X}+\epsilon \mathbf{Z}\rfloor)=\infty$ for every $\epsilon \geq 0$.

(ii) If $H(\lfloor\mathbf{X}\rfloor)<\infty$ and $\operatorname{Pr}\left(\mathbf{X} \in \mathbb{Z}^{d}\right)=0$, then

$$
\lim _{\epsilon \downarrow 0} H(\lfloor\mathbf{X}+\epsilon \mathbf{Z}\rfloor)=H(\lfloor\mathbf{X}\rfloor) .
$$

Proof: See appendix.

The random vector $\mathbf{Z}_{D}$ is independent of $\mathbf{X}$ and has the same pdf as $D^{1 / r} \mathbf{Z}_{1}$, where $\mathbf{Z}_{1}$ satisfies $\mathrm{E}\left[\left\|\mathbf{Z}_{1}\right\|^{r}\right]=1$. Furthermore, by assumption, $H(\lfloor\mathbf{X}\rfloor)<\infty$ and $\operatorname{Pr}\left(\mathbf{X} \in \mathbb{Z}^{d}\right)=0$ (since $\mathbf{X}$ has a pdf and $\mathbb{Z}^{d}$ is countable). It thus follows from Part (ii) of Lemma 1 that

$$
\begin{aligned}
\lim _{D \downarrow 0} H\left(\left\lfloor\mathbf{X}+\mathbf{Z}_{D}\right\rfloor\right) & =\lim _{D \downarrow 0} H\left(\left\lfloor\mathbf{X}+D^{1 / r} \mathbf{Z}_{1}\right\rfloor\right) \\
& =H(\lfloor\mathbf{X}\rfloor) .
\end{aligned}
$$

Combining (29) with (27) yields (21), which in turn demonstrates that the Shannon lower bound is asymptotically tight if $H(\lfloor\mathbf{X}\rfloor)<\infty$ and $|h(\mathbf{X})|<\infty$. This proves Theorem 2 .

\section{Proof of Theorem 3}

To show that $H(\lfloor\mathbf{X}\rfloor)<\infty$ implies $R(D)<\infty$ for every $D>0$, we upper-bound the rate-distortion function by choosing $\hat{\mathbf{X}}$ in (2) as

$$
\hat{\mathbf{X}}=\frac{\lfloor m \mathbf{X}\rfloor}{m}, \quad \text { for some positive integer } m .
$$

For $m$ sufficiently large, this choice satisfies the distortion constraint (3). Indeed, on a finite-dimensional vector space any two norms are within a constant factor of one another [25, Cor. 5.4 .5 , p. 272], so

$$
\underline{c}\|\mathbf{z}\|_{1} \leq\|\mathbf{z}\| \leq \bar{c}\|\mathbf{z}\|_{1}, \quad \mathbf{z} \in \mathbb{R}^{d}
$$

for some constants $\bar{c} \geq \underline{c}>0$, where

$$
\|\mathbf{z}\|_{1} \triangleq\left|z_{1}\right|+\ldots+\left|z_{d}\right|, \quad \mathbf{z}=\left(z_{1}, \ldots, z_{d}\right) \in \mathbb{R}^{d}
$$

denotes the $L_{1}$-norm. Since each component of $|\mathbf{X}-\hat{\mathbf{X}}|$ is upper-bounded by $1 / m$, we thus have that $\|\mathbf{X}-\hat{\mathbf{X}}\| \leq \bar{c} d / m$. Hence,

$$
\mathrm{E}\left[\|\mathbf{X}-\hat{\mathbf{X}}\|^{r}\right] \leq D, \quad \text { for } m \geq \frac{\bar{c} d}{D^{1 / r}}
$$

It follows that, for $m \geq \bar{c} d / D^{1 / r}$,

$$
R(D) \leq H(\hat{\mathbf{X}})=H\left(\frac{\lfloor m \mathbf{X}\rfloor}{m}\right) .
$$

Furthermore, by generalizing (11) in [16] to the vector case, we obtain

$$
H\left(\frac{\lfloor m \mathbf{X}\rfloor}{m}\right) \leq H(\lfloor\mathbf{X}\rfloor)+d \log m .
$$

Thus, $H(\lfloor\mathbf{X}\rfloor)<\infty$ implies that $R(D)<\infty$ for every $D>0$.

To prove that $H(\lfloor\mathbf{X}\rfloor)=\infty$ implies $R(D)=\infty$ for every $D>0$, we show that $I(\mathbf{X} ; \hat{\mathbf{X}})=\infty$ for every pair of random vectors $(\mathbf{X}, \hat{\mathbf{X}})$ satisfying (3) and $H(\lfloor\mathbf{X}\rfloor)=\infty$. To this end, we follow along the lines of the proof of Theorem 6 in [20, App. A]. Indeed, it follows from the data processing inequality [26, Cor. 7.16] that for any arbitrary $\Upsilon>0$

$$
I(\mathbf{X} ; \hat{\mathbf{X}}) \geq I\left(g_{\Upsilon}(\lfloor\mathbf{X}\rfloor) ;\lfloor\hat{\mathbf{X}}\rfloor\right)
$$

where the function $g_{\Upsilon}: \mathbb{R}^{d} \rightarrow[-\Upsilon, \Upsilon]^{d}$ clips its argument to the hypercube $[-\Upsilon, \Upsilon]^{d}$, i.e.,

$$
g_{\Upsilon}(\mathbf{x}) \triangleq \max \{\min \{\mathbf{x}, \mathbf{\Upsilon}\},-\Upsilon \mathbf{\Upsilon}\}, \quad \mathbf{x} \in \mathbb{R}^{d} .
$$

In (36), $\Upsilon$ denotes the $d$-dimensional vector $(\Upsilon, \ldots, \Upsilon)$, and $\max \{\cdot, \cdot\}$ and $\min \{\cdot, \cdot\}$ denote the component-wise maximum and minimum, respectively. Since $H\left(g_{\Upsilon}(\lfloor\mathbf{X}\rfloor)\right)$ is finite, the mutual information on the right-hand side (RHS) of (35) can be written in the form

$$
\begin{aligned}
I\left(g_{\Upsilon}(\lfloor\mathbf{X}\rfloor) ;\lfloor\hat{\mathbf{X}}\rfloor\right)= & H\left(g_{\Upsilon}(\lfloor\mathbf{X}\rfloor)\right) \\
& -H(g \Upsilon(\lfloor\mathbf{X}\rfloor) \mid\lfloor\hat{\mathbf{X}}\rfloor)
\end{aligned}
$$

which is well-defined.

We first show that the second entropy on the RHS of (37) is bounded in $\Upsilon$ for every pair of vectors $(\mathbf{X}, \hat{\mathbf{X}})$ satisfying (3). Using basic properties of entropy together with the fact that the entropy of a function of a random variable is less than or equal to the entropy of the random variable itself [21, Ex. 5, p. 43], we obtain

$$
\begin{aligned}
H\left(g_{\Upsilon}(\lfloor\mathbf{X}\rfloor) \mid\lfloor\hat{\mathbf{X}}\rfloor\right) \leq & H(\lfloor\mathbf{X}-\hat{\mathbf{X}}\rfloor) \\
& +H(\lfloor\mathbf{X}\rfloor \mid\lfloor\hat{\mathbf{X}}\rfloor,\lfloor\mathbf{X}-\hat{\mathbf{X}}\rfloor) .
\end{aligned}
$$

Since $\mathbf{E}[\log (1+\|\mathbf{X}-\hat{\mathbf{X}}\|)]<\infty$ for all $(\mathbf{X}, \hat{\mathbf{X}})$ satisfying (3), generalizing Proposition 1 in [18] to the vector case yields that

$$
H(\lfloor\mathbf{X}-\hat{\mathbf{X}}\rfloor)<\infty .
$$

Furthermore, denoting $\mathbf{Y}=\mathbf{X}-\hat{\mathbf{X}}$, we obtain

$$
\begin{aligned}
H(\lfloor\mathbf{X}\rfloor \mid\lfloor\hat{\mathbf{X}}\rfloor,\lfloor\mathbf{X}-\hat{\mathbf{X}}\rfloor) & =H(\lfloor\hat{\mathbf{X}}+\mathbf{Y}\rfloor \mid\lfloor\hat{\mathbf{X}}\rfloor,\lfloor\mathbf{Y}\rfloor) \\
& \leq d \log 2
\end{aligned}
$$

since, given $\lfloor\hat{\mathbf{X}}\rfloor$ and $\lfloor\mathbf{Y}\rfloor$, each component of $\lfloor\hat{\mathbf{X}}+\mathbf{Y}\rfloor$ can only take on the values $\left\lfloor\hat{X}_{\ell}\right\rfloor+\left\lfloor Y_{\ell}\right\rfloor$ and $\left\lfloor\hat{X}_{\ell}\right\rfloor+\left\lfloor Y_{\ell}\right\rfloor+1$. Combining (38)-(40) yields

$$
\sup _{\Upsilon>0} H(g \Upsilon(\lfloor\mathbf{X}\rfloor) \mid\lfloor\hat{\mathbf{X}}\rfloor)<\infty .
$$

We next show that if $H(\lfloor\mathbf{X}\rfloor)=\infty$, then

$$
\lim _{\Upsilon \rightarrow \infty} H\left(g_{\Upsilon}(\lfloor\mathbf{X}\rfloor)\right)=\infty .
$$


Since $\Upsilon>0$ is arbitrary, it then follows from (35) and (37) that

$$
I(\mathbf{X} ; \hat{\mathbf{X}}) \geq \varlimsup_{\Upsilon \rightarrow \infty}\left\{H\left(g_{\Upsilon}(\lfloor\mathbf{X}\rfloor)\right)-H\left(g_{\Upsilon}(\lfloor\mathbf{X}\rfloor) \mid\lfloor\hat{\mathbf{X}}\rfloor\right)\right\}
$$

which by (41) and (42) is infinite. Hence, $I(\mathbf{X} ; \hat{\mathbf{X}})=\infty$ for every pair of random vectors $(\mathbf{X}, \hat{\mathbf{X}})$ satisfying (3) and $H(\lfloor\mathbf{X}\rfloor)=\infty$, which implies that the rate-distortion function $R(D)$ is infinite for every $D>0$.

To prove (42), we note that

$$
\begin{aligned}
& H\left(g_{\Upsilon}(\lfloor\mathbf{X}\rfloor)\right) \\
& \quad \geq \sum_{\mathbf{i} \in \mathbb{Z}^{d} \cap(-\Upsilon, \Upsilon)^{d}} \operatorname{Pr}(\lfloor\mathbf{X}\rfloor=\mathbf{i}) \log \frac{1}{\operatorname{Pr}(\lfloor\mathbf{X}\rfloor=\mathbf{i})}
\end{aligned}
$$

because $\operatorname{Pr}\left(g_{\Upsilon}(\lfloor\mathbf{X}\rfloor)=\mathbf{i}\right) \log \left(1 / \operatorname{Pr}\left(g_{\Upsilon}(\lfloor\mathbf{X}\rfloor)=\mathbf{i}\right)\right) \geq 0$ for $\mathbf{i} \notin(-\Upsilon, \Upsilon)^{d}$ and $\operatorname{Pr}\left(g_{\Upsilon}(\lfloor\mathbf{X}\rfloor)=\mathbf{i}\right)=\operatorname{Pr}(\lfloor\mathbf{X}\rfloor=\mathbf{i})$ for $\mathbf{i} \in(-\Upsilon, \Upsilon)^{d}$. Since the summands in (44) are nonnegative, the RHS of (44) converges to $H(\lfloor\mathbf{X}\rfloor)$ as $\Upsilon$ tends to infinity. This proves (42) and concludes the proof of Theorem 3.

\section{Conclusions}

The Shannon lower bound is one of the few lower bounds on the rate-distortion function that hold for a large class of sources. We have demonstrated that this lower bound is asymptotically tight as the allowed distortion vanishes for all sources having finite differential entropy and finite Rényi information dimension. Conversely, we have demonstrated that if the source has infinite Rényi information dimension, then the rate-distortion function is infinite for any finite distortion level.

Assuming finite Rényi information dimension is tantamount to assuming that quantizing the source with a cubic lattice quantizer of unit-volume cells gives rise to a discrete random vector of finite entropy. The latter assumption is natural in ratedistortion theory and often encountered. To this effect, we have demonstrated that this assumption is not only natural, but it is also a necessary and sufficient condition for the asymptotic tightness of the Shannon lower bound.

For ease of exposition, we have only considered norm-based difference distortion measures, which is less general than the distortion measures studied, e.g., by Linder and Zamir in [7]. While our analysis could probably be generalized to more general distortion measures, we have refrained from doing so, because we believe that it would obscure the analysis without offering much more insight.

\section{APPENDIX}

\section{A. Proof of Lemma 1: Part (i)}

We shall show by contradiction that if $H(\lfloor\mathbf{X}\rfloor)=\infty$, then $H(\lfloor\mathbf{X}+\epsilon \mathbf{Z}\rfloor)=\infty$ for every $\epsilon \geq 0$. So let us assume that $H(\lfloor\mathbf{X}\rfloor)=\infty$, but that there exists an $\epsilon \geq 0$ for which $H(\lfloor\mathbf{X}+\epsilon \mathbf{Z}\rfloor)<\infty$. It then follows that, for any arbitrary $\Upsilon>0$, the difference $H(\lfloor\mathbf{X}+\epsilon \mathbf{Z}\rfloor)-H\left(\lfloor\mathbf{X}+\epsilon \mathbf{Z}\rfloor \mid g_{\Upsilon}(\lfloor\mathbf{X}\rfloor)\right)$ is well-defined and equal to $I\left(\lfloor\mathbf{X}+\epsilon \mathbf{Z}\rfloor ; g_{\Upsilon}(\lfloor\mathbf{X}\rfloor)\right)$. (The function $g_{\Upsilon}(\cdot)$ has been defined in (36).) Consequently, by the nonnegativity of entropy,

$$
H(\lfloor\mathbf{X}+\epsilon \mathbf{Z}\rfloor) \geq I\left(\lfloor\mathbf{X}+\epsilon \mathbf{Z}\rfloor ; g_{\Upsilon}(\lfloor\mathbf{X}\rfloor)\right) .
$$

Furthermore, $H\left(g_{\Upsilon}(\lfloor\mathbf{X}\rfloor)\right)$ is finite, so the mutual information on the RHS of (45) can also be written as

$$
\begin{aligned}
I\left(\lfloor\mathbf{X}+\epsilon \mathbf{Z}\rfloor ; g_{\Upsilon}(\lfloor\mathbf{X}\rfloor)\right)= & H\left(g_{\Upsilon}(\lfloor\mathbf{X}\rfloor)\right) \\
& -H\left(g_{\Upsilon}(\lfloor\mathbf{X}\rfloor) \mid\lfloor\mathbf{X}+\epsilon \mathbf{Z}\rfloor\right) .
\end{aligned}
$$

We next show that

$$
\sup _{\Upsilon>0} H(g \Upsilon(\lfloor\mathbf{X}\rfloor) \mid\lfloor\mathbf{X}+\epsilon \mathbf{Z}\rfloor)<\infty .
$$

To this end, we follow the steps (38)-(40) in Section IV. Indeed, as in (38), it can be shown that

$$
\begin{aligned}
& H\left(g_{\Upsilon}(\lfloor\mathbf{X}\rfloor) \mid\lfloor\mathbf{X}+\epsilon \mathbf{Z}\rfloor\right) \\
& \quad \leq H(\lfloor\epsilon \mathbf{Z}\rfloor)+H(\lfloor\mathbf{X}\rfloor \mid\lfloor\mathbf{X}+\epsilon \mathbf{Z}\rfloor,\lfloor\epsilon \mathbf{Z}\rfloor) .
\end{aligned}
$$

Generalizing Proposition 1 in [18] to the vector case then yields that the first entropy on the RHS of (48) is finite, since the lemma's assumption $\mathrm{E}\left[\|\mathbf{Z}\|^{r}\right]<\infty$ implies that $E[\log (1+\|\epsilon \mathbf{Z}\|)]<\infty$. Moreover, following the steps in (40), the second entropy on the RHS of (48) can be upper-bounded by

$$
H(\lfloor\mathbf{X}\rfloor \mid\lfloor\mathbf{X}+\epsilon \mathbf{Z}\rfloor,\lfloor\epsilon \mathbf{Z}\rfloor) \leq d \log 2 .
$$

The claim (47) thus follows.

Since $\Upsilon>0$ is arbitrary, (45) and (46) give

$$
\begin{aligned}
& H(\lfloor\mathbf{X}+\epsilon \mathbf{Z}\rfloor) \geq \varlimsup_{\Upsilon \rightarrow \infty}\left\{H\left(g_{\Upsilon}(\lfloor\mathbf{X}\rfloor)\right)\right. \\
&\left.-H\left(g_{\Upsilon}(\lfloor\mathbf{X}\rfloor) \mid\lfloor\mathbf{X}+\epsilon \mathbf{Z}\rfloor\right)\right\} .
\end{aligned}
$$

However, if $H(\lfloor\mathbf{X}\rfloor)=\infty$ then, by (42) and (47), the RHS of (50) is infinite, which contradicts the assumption that there exists an $\epsilon \geq 0$ for which $H(\lfloor\mathbf{X}+\epsilon \mathbf{Z}\rfloor)<\infty$. This proves Part (i) of Lemma 1.

\section{B. Proof of Lemma 1: Part (ii)}

Using basic properties of entropy, we obtain

$$
\begin{aligned}
H(\lfloor\mathbf{X}+\epsilon \mathbf{Z}\rfloor) & \leq H(\lfloor\mathbf{X}\rfloor)+H(\lfloor\mathbf{X}+\epsilon \mathbf{Z}\rfloor \mid\lfloor\mathbf{X}\rfloor) \\
& \leq H(\lfloor\mathbf{X}\rfloor)+H\left(\mathbf{V}_{\epsilon}\right)
\end{aligned}
$$

and

$$
\begin{aligned}
H(\lfloor\mathbf{X}+\epsilon \mathbf{Z}\rfloor) & \geq H(\lfloor\mathbf{X}\rfloor)-H(\lfloor\mathbf{X}\rfloor \mid\lfloor\mathbf{X}+\epsilon \mathbf{Z}\rfloor) \\
& \geq H(\lfloor\mathbf{X}\rfloor)-H\left(\mathbf{V}_{\epsilon}\right)
\end{aligned}
$$

where we define $\mathbf{V}_{\epsilon} \triangleq\lfloor\mathbf{X}+\epsilon \mathbf{Z}\rfloor-\lfloor\mathbf{X}\rfloor$. Note that $\mathbf{V}_{\epsilon}$ can also be written as $\mathbf{V}_{\epsilon}=\lfloor\overline{\mathbf{X}}+\epsilon \mathbf{Z}\rfloor$, where $\overline{\mathbf{X}} \triangleq \mathbf{X}-\lfloor\mathbf{X}\rfloor$.

In view of (51) and (52), Part (ii) of Lemma 1 follows by showing that $H\left(\mathbf{V}_{\epsilon}\right)$ vanishes as $\epsilon$ tends to zero. We begin by writing this entropy as (see, e.g., [9, Eq. (81)])

$$
H\left(\mathbf{V}_{\epsilon}\right)=h(\lfloor\overline{\mathbf{X}}+\epsilon \mathbf{Z}\rfloor+\mathbf{U})
$$

where $\mathbf{U}$ is a $d$-dimensional random vector that is uniformly distributed over the hypercube $[0,1)^{d}$ and that is independent of $(\mathbf{X}, \mathbf{Z})$. We next show that

$$
\lim _{\epsilon \downarrow 0} h(\lfloor\overline{\mathbf{X}}+\epsilon \mathbf{Z}\rfloor+\mathbf{U})=h(\mathbf{U}) .
$$


The differential entropy of $\mathbf{U}$ is zero, so (53) and (54) demonstrate that $H\left(\mathbf{V}_{\epsilon}\right)$ vanishes as $\epsilon$ tends to zero, thereby proving Part (ii) of Lemma 1.

Since conditioning reduces entropy, we have

$$
h(\lfloor\overline{\mathbf{X}}+\epsilon \mathbf{Z}\rfloor+\mathbf{U}) \geq h(\mathbf{U}) .
$$

To prove (54), it thus remains to show that

$$
\varlimsup_{\epsilon \downarrow 0} h(\lfloor\overline{\mathbf{X}}+\epsilon \mathbf{Z}\rfloor+\mathbf{U}) \leq h(\mathbf{U}) .
$$

To this end, we follow along the lines of the proof of Theorem 1 in [7] (see also the proof of Lemma 6.9 in [27]). Let the random vectors $\tilde{\mathbf{Y}}_{\epsilon}$ and $\tilde{\mathbf{Y}}_{0}$ have the respective pdfs

$$
\begin{aligned}
& f_{\tilde{\mathbf{Y}}_{\epsilon}}(\mathbf{y})=\left(\frac{r}{d}\right)^{\frac{d}{r}-1} \frac{1}{V_{d} \Gamma(d / r) \sigma_{\epsilon}^{\frac{d}{r}}} e^{-\frac{d}{r \sigma_{\epsilon}}\|\mathbf{y}\|^{r}} \\
& f_{\tilde{\mathbf{Y}}_{0}}(\mathbf{y})=\left(\frac{r}{d}\right)^{\frac{d}{r}-1} \frac{1}{V_{d} \Gamma(d / r) \mathrm{E}\left[\|\mathbf{U}\|^{r}\right]^{\frac{d}{r}}} e^{-\frac{d}{r E[\|\mathbf{U}\| r]}\|\mathbf{y}\|^{r}}
\end{aligned}
$$

for $\mathbf{y} \in \mathbb{R}^{d}$, where

$$
\sigma_{\epsilon} \triangleq \mathbf{E}\left[\|\lfloor\overline{\mathbf{X}}+\epsilon \mathbf{Z}\rfloor+\mathbf{U}\|^{r}\right] .
$$

It follows that

$$
\begin{array}{r}
D\left(f_{\lfloor\overline{\mathbf{X}}+\epsilon \mathbf{Z}\rfloor+\mathbf{U}} \| f_{\tilde{\mathbf{Y}}_{\epsilon}}\right)=\frac{d}{r}+\log \left(\frac{V_{d} \Gamma(d / r)}{(r / d)^{d / r-1}}\right) \\
+\frac{d}{r} \log \sigma_{\epsilon}-h(\lfloor\overline{\mathbf{X}}+\epsilon \mathbf{Z}\rfloor+\mathbf{U})
\end{array}
$$

and

$$
\begin{aligned}
D\left(f_{\mathbf{U}} \| f_{\tilde{\mathbf{Y}}_{0}}\right)= & \frac{d}{r}+\log \left(\frac{V_{d} \Gamma(d / r)}{(r / d)^{d / r-1}}\right) \\
& +\frac{d}{r} \log \mathbf{E}\left[\|\mathbf{U}\|^{r}\right]-h(\mathbf{U}) .
\end{aligned}
$$

As we shall argue next, the pdf of $\lfloor\overline{\mathbf{X}}+\epsilon \mathbf{Z}\rfloor+\mathbf{U}$ converges pointwise to the pdf of $\mathbf{U}$ as $\epsilon$ tends to zero, so by Scheffe's lemma $\lfloor\overline{\mathbf{X}}+\epsilon \mathbf{Z}\rfloor+\mathbf{U} \Rightarrow \mathbf{U}$ as $\epsilon$ tends to zero. Indeed, the pdf of $\lfloor\overline{\mathbf{X}}+\epsilon \mathbf{Z}\rfloor+\mathbf{U}$ is given by

$$
f_{\lfloor\overline{\mathbf{X}}+\epsilon \mathbf{Z}\rfloor+\mathbf{U}}(\mathbf{x})=\sum_{\mathbf{i} \in \mathbb{Z}^{d}} \operatorname{Pr}(\lfloor\overline{\mathbf{X}}+\epsilon \mathbf{Z}\rfloor=\mathbf{i}) \mathbf{1}\{\lfloor\mathbf{x}\rfloor=\mathbf{i}\}
$$

for $\mathbf{x} \in \mathbb{R}^{d}$. Since $\mathbf{E}\left[\|\mathbf{Z}\|^{r}\right]<\infty$, we have that $\epsilon \mathbf{Z} \rightarrow \mathbf{0}$ almost surely as $\epsilon$ tends to zero, which implies that $\epsilon \mathbf{Z} \Rightarrow \mathbf{0}$ as $\epsilon$ tends to zero. Furthermore, the independence of $\mathbf{X}$ and $\mathbf{Z}$ implies that $\overline{\mathbf{X}}+\epsilon \mathbf{Z} \Rightarrow \overline{\mathbf{X}}$ as $\epsilon$ tends to zero. Since by assumption $\operatorname{Pr}\left(\mathbf{X} \in \mathbb{Z}^{d}\right)=0$, it follows that the probability $\operatorname{Pr}\left(\overline{\mathbf{X}} \in \mathbb{Z}^{d}\right)$ is zero, so [22, Th. 2.8.1, p. 122]

$$
\lim _{\epsilon \downarrow 0} \operatorname{Pr}(\lfloor\overline{\mathbf{X}}+\epsilon \mathbf{Z}\rfloor=\mathbf{i})=\operatorname{Pr}(\lfloor\overline{\mathbf{X}}\rfloor=\mathbf{i})=\mathbf{1}\{\mathbf{i}=\mathbf{0}\}
$$

for $\mathbf{i} \in \mathbb{Z}^{d}$, where the last step follows because, by definition, $\lfloor\overline{\mathbf{X}}\rfloor=\mathbf{0}$ almost surely. Applying (62) to (61), and noting that

$$
f_{\mathbf{U}}(\mathbf{u})=\mathbf{1}\{\lfloor\mathbf{u}\rfloor=\mathbf{0}\}, \quad \mathbf{u} \in \mathbb{R}^{d}
$$

the claim that $f_{[\overline{\mathbf{X}}+\epsilon \mathbf{Z}\rfloor+\mathbf{U}}$ converges pointwise to $f_{\mathbf{U}}$ as $\epsilon$ tends to zero follows.

We next show that

$$
\lim _{\epsilon \downarrow 0} \mathrm{E}\left[\|\lfloor\overline{\mathbf{X}}+\epsilon \mathbf{Z}\rfloor+\mathbf{U}\|^{r}\right]=\mathrm{E}\left[\|\mathbf{U}\|^{r}\right] .
$$

Since $f_{\tilde{\mathbf{Y}}_{\epsilon}}$ is a continuous function of $\sigma_{\epsilon}$, this implies that $f_{\tilde{\mathbf{Y}}_{\epsilon}}$ converges pointwise to $f_{\tilde{\mathbf{Y}}_{0}}$ as $\epsilon$ tends to zero, so by Scheffe's lemma $\tilde{\mathbf{Y}}_{\epsilon} \Rightarrow \tilde{\mathbf{Y}}_{0}$ as $\epsilon$ tends to zero.

To prove (64), we first note that, since the function $x \mapsto\lfloor x\rfloor$ is continuous for $x \notin \mathbb{Z}$, and since on a finite-dimensional vector space component-wise convergence is equivalent to convergence with respect to $\|\cdot\|[25$, p. 273], we have

$$
\lim _{\epsilon \downarrow 0}\|\lfloor\overline{\mathbf{x}}+\epsilon \mathbf{z}\rfloor+\mathbf{u}\|^{r}=\|\lfloor\overline{\mathbf{x}}\rfloor+\mathbf{u}\|^{r}=\|\mathbf{u}\|^{r}
$$

for every $\mathbf{z} \in \mathbb{R}^{d}$ and $\mathbf{u} \in[0,1)^{d}$, and for $\overline{\mathbf{x}} \in(0,1)^{d}$. Furthermore, for every $0 \leq \epsilon \leq 1$,

$$
\begin{aligned}
\|\lfloor\overline{\mathbf{x}}+\epsilon \mathbf{z}\rfloor+\mathbf{u}\|^{r} & \leq \bar{c}^{r}\|\lfloor\overline{\mathbf{x}}+\epsilon \mathbf{z}\rfloor+\mathbf{u}\|_{1}^{r} \\
& \leq \bar{c}^{r}\||\mathbf{z}|+\mathbf{3}\|_{1}^{r} \\
& \leq \bar{c}^{r}\left(\|\mathbf{z}\|_{1}+3 d\right)^{r} \\
& \leq \frac{\bar{c}^{r}}{\underline{c}^{r}}(\|\mathbf{z}\|+3 \underline{c} d)^{r}
\end{aligned}
$$

where 3 denotes the $d$-dimensional vector $(3, \ldots, 3)$ and $\underline{c}$ and $\bar{c}$ are as in (31). Here the first step follows from (31); the second step follows because $|\lfloor x\rfloor| \leq|x|+1, x \in \mathbb{R}$ and because the components of $\overline{\mathbf{x}}$ and $\mathbf{u}$ satisfy $0 \leq \bar{x}_{\ell}, u_{\ell}<1$; the third step follows from the triangle inequality and because $\|\mathbf{3}\|_{1}=3 d$; and the last step follows again from (31).

The lemma's assumptions $\mathbf{E}\left[\|\mathbf{Z}\|^{r}\right]<\infty$ and $\operatorname{Pr}\left(\mathbf{X} \in \mathbb{Z}^{d}\right)=$ 0 imply that

$$
\mathrm{E}\left[\frac{\bar{c}^{r}}{\underline{c}^{r}}(\|\mathbf{Z}\|+3 \underline{c} d)^{r}\right]<\infty
$$

and

$$
\operatorname{Pr}\left(\overline{\mathbf{X}} \in(0,1)^{d}\right)=1
$$

respectively. Consequently, (64) follows from (65) and the dominated convergence theorem [22, Th. 1.6.9, p. 50].

We conclude that $\lfloor\overline{\mathbf{X}}+\epsilon \mathbf{Z}\rfloor+\mathbf{U} \Rightarrow \mathbf{U}$ and $\tilde{\mathbf{Y}}_{\epsilon} \Rightarrow \tilde{\mathbf{Y}}_{0}$ as $\epsilon$ tends to zero, so the lower semicontinuity of relative entropy gives

$$
\varliminf_{\epsilon \downarrow} D\left(f_{\lfloor\overline{\mathbf{X}}+\epsilon \mathbf{Z}\rfloor+\mathbf{U}} \| f_{\tilde{\mathbf{Y}}_{\epsilon}}\right) \geq D\left(f_{\mathbf{U}} \| f_{\tilde{\mathbf{Y}}_{0}}\right) .
$$

Combining (69) with (59) and (60), it follows that

$$
\begin{aligned}
\varlimsup_{\epsilon \downarrow 0}\left\{\frac{d}{r} \log \sigma_{\epsilon}\right. & h(\lfloor\overline{\mathbf{X}}+\epsilon \mathbf{Z}\rfloor+\mathbf{U})\} \\
& \geq \frac{d}{r} \log \mathbf{E}\left[\|\mathbf{U}\|^{r}\right]-h(\mathbf{U})
\end{aligned}
$$

which together with (64) proves (56). This concludes the proof of Part (ii) of Lemma 1.

\section{ACKNOWLEDGMENT}

The author wishes to thank Helmut Bölcskei, Tamás Linder, David Stotz, and Gonzalo Vazquez-Vilar for helpful discussions. The author further wishes to thank Giuseppe Durisi and Tamás Linder for calling his attention to references [18] and [19], respectively. 


\section{REFERENCES}

[1] C. E. Shannon, "Coding theorems for a discrete source with a fidelity criterion," IRE International Convention Record, vol. 7, pp. 142-163, 1959.

[2] T. Berger, Rate Distortion Theory: Mathematical Basis for Data Compression, ser. Electrical Engineering Series. Prentice Hall, 1971.

[3] Y. Yamada, S. Tazaki, and R. M. Gray, "Asymptotic performance of block quantizers with difference distortion measures," IEEE Trans. Inform. Theory, vol. 26, pp. 6-14, Jan. 1980.

[4] Y. N. Linkov, "Evaluation of epsilon entropy of random variables for small epsilon," Problemy Peredachi Informatsii (Problems of Inform. Transm.), vol. 1, pp. 12-18, 1965.

[5] A. M. Gerrish and P. M. Schultheiss, "Information rates of non-Gaussian processes," IEEE Trans. Inform. Theory, vol. 10, pp. 265-271, Oct. 1964.

[6] J. Binia, M. Zakai, and J. Ziv, "On the $\epsilon$-entropy and the rate-distortion function of certain non-Gaussian process," IEEE Trans. Inform. Theory, vol. 20, pp. 514-524, July 1974.

[7] T. Linder and R. Zamir, "On the asymptotic tightness of the Shannon lower bound," IEEE Trans. Inform. Theory, vol. 40, no. 6, pp. 20262031, Nov. 1994.

[8] V. Kostina, "Data compression with low distortion and finite blocklength," in Proc. 53rd Allerton Conf. Comm., Contr. and Comp., Allerton H., Monticello, Il, Sep. 30 - Oct. 2, 2015.

[9] —-, "Data compression with low distortion and finite blocklength," Jan. 2016. [Online]. Available: http://arxiv.org/pdf/1510.02190v2

[10] H. Gish and J. N. Pierce, "Asymptotically efficient quantizing," IEEE Trans. Inform. Theory, vol. 14, no. 5, pp. 676-683, Sept. 1968.

[11] R. M. Gray and D. L. Neuhoff, "Quantization," IEEE Trans. Inform. Theory, vol. 44, no. 6, pp. 2325-2383, Oct. 1998.

[12] P. L. Zador, "Topics in the asymptotic quantization of continuous random variables," Bell Laboratories, Tech. Rep., 1966.

[13] R. M. Gray, T. Linder, and J. Li, "A Langrangian formulation of Zador's entropy-constrained quantization theorem," IEEE Trans. Inform. Theory, vol. 28, no. 3, pp. 695-707, Mar. 2002.

[14] T. Koch and G. Vazquez-Vilar, "Rate-distortion bounds for highresolution vector quantization via Gibbs's inequality," July 2015. [Online]. Available: http://arxiv.org/abs/1507.08349
[15] — "A general rate-distortion converse bound for entropy-constrained scalar quantization," in Proc. IEEE Int. Symposium on Inf. Theory, Barcelona, Spain, July 10-15, 2016.

[16] A. Rényi, "On the dimension and entropy of probability distributions," Acta Mathematica Hungarica, vol. 10, no. 1-2, Mar. 1959.

[17] T. Kawabata and A. Dembo, "The rate-distortion dimension of sets and measures," IEEE Trans. Inform. Theory, vol. 40, no. 5, pp. 1564-1572, Sept. 1994.

[18] Y. Wu and S. Verdú, "Rényi information dimension: Fundamental limits of almost lossless analog compression," IEEE Trans. Inform. Theory, vol. 56, no. 8, pp. 3721-3748, Aug. 2010.

[19] I. Csiszár, "Some remarks on the dimension and entropy of random variables," Acta Mathematica Hungarica, vol. 12, no. 3-4, pp. 399-408, Sept. 1961.

[20] D. Stotz and H. Bölcskei, "Degrees of freedom in vector interference channels," IEEE Trans. Inform. Theory, vol. 62, no. 7, pp. 4172-4197, July 2016.

[21] T. M. Cover and J. A. Thomas, Elements of Information Theory, 1st ed. John Wiley \& Sons, 1991.

[22] R. B. Ash and C. A. Doléans-Dade, Probability and Measure Theory, 2nd ed. Elsevier/Academic Press, 2000.

[23] P. Billingsley, Probability and Measure, 3rd ed., ser. Wiley Series in Probability and Mathematical Statistics: Probability and Mathematical Statistics. John Wiley \& Sons, 1995.

[24] I. Csiszár, "Arbitrarily varying channel with general alphabets and states," IEEE Trans. Inform. Theory, vol. 38, no. 6, pp. 1725-1742, Nov. 1992.

[25] R. A. Horn and C. R. Johnson, Matrix Analysis. Cambridge University Press, 1985.

[26] R. M. Gray, Entropy and Information Theory, 2nd ed. Springer Verlag, 2011.

[27] A. Lapidoth and S. M. Moser, "Capacity bounds via duality with applications to multiple-antenna systems on flat fading channels," IEEE Trans. Inform. Theory, vol. 49, no. 10, pp. 2426-2467, Oct. 2003.

Tobias Koch (S'02, M'09) received the M.Sc. (with distinction) and Ph.D degrees in electrical engineering from ETH Zurich, Switzerland, in 2004 and 2009, respectively.

He is a Visiting Professor and a Ramón y Cajal Research Fellow at the Department of Signal Theory and Communications, Universidad Carlos III de Madrid (UC3M), Leganés, Spain. From June 2010 to May 2012, he was a Marie Curie Intra-European Research Fellow with the University of Cambridge, Cambridge, U.K. He was also a Research Intern at Bell Labs, Murray Hill, NJ, USA, in 2004, and the Universitat Pompeu Fabra (UPF), Barcelona, Spain, in 2007. He joined UC3M in 2012. His research interests include digital communication theory and information theory.

Dr. Koch is serving as the Vice Chair of the Spain Chapter of the IEEE Information Theory Society in 2013-2016. He was the recipient of a Ramón y Cajal Research Fellowship, a Marie Curie Intra-European Fellowship, a Marie Curie Career Integration Grant, and a Fellowship for Prospective Researchers from the Swiss National Science Foundation. 